\title{
THE RELATIONSHIP BETWEEN CONCENTRATION OF SULFAMERAZINE IN BODY FLUIDS AND THE RESPONSE IN TREATMENT OF MENINGOCOCCIC MENINGITIS ${ }^{1}$
}

\author{
BY JOHN G. REINHOLD, HARRISON F. FLIPPIN, JOSEPH J. ZIMMERMAN, \\ WILLIAM I. GEFTER AND JOHN G. RIDDLER, WITH THE TECHNICAL \\ ASSISTANCE OF LILLIAN POLLACK, ROSE P. FELDMAN, \\ AND RITA M. FENWICK \\ (From the Committee on Chemotherapy and the Division of Biochemistry, The Laboratories, \\ Philadelphia General Hospital, Philadelphia)
}

(Received for publication September 25, 1944)

Numerous studies of bacteriostatic activity of sulfanilamide-type compounds indicate that for effective action a minimal concentration must be attained and that, in general, rising concentration is accompanied by increased activity. Therapeutic trials in experimental animals have led to a similar conclusion. However, attempts to correlate the response of the patient with the concentration of the sulfonamide in the body fluids have not met with much success. It is not surprising, therefore, that divergence of opinion exists as to the importance of this aspect of sulfonamide therapy. The belief widely held among clinicians and reflected in some reviews (1) is that the concentration of sulfonamide in blood is not of critical significance in treatment of many infections.

However, there is general agreement that concentration of sulfonamide in body fluids is a factor of considerable importance in the treatment of meningococcic meningitis, a view shared by those (1) who question the importance of measurement of drug concentration in other infections. Recent reviews $(2,3)$ have advocated the maintenance in meningitis therapy of blood levels of 10 to $15 \mathrm{mgm}$. per $100 \mathrm{ml}$. for sulfanilamide or sulfadiazine. Lower levels have been found to give satisfactory results when sulfathiazole was used (4).

It is the purpose of the present paper to evaluate the response of patients suffering from meningococcic meningitis to treatment with a highly effective sulfonamide, sulfamerazine (methyl sulfadiazine, 2-sulfanilamido-4-methyl-pyrimidine), with particular reference to the concentration of this

1 This work was aided by grants from Sharp and Dohme Laboratories, Glenolden, $\mathrm{Pa}$. and from The $\mathrm{Re}$ search Fund for Infectious Diseases, University of Pennsylvania, Philadelphia. substance in body fluids. The comparative significance of drug concentrations in plasma, plasma ultrafiltrate, and in cerebrospinal fluid also has been examined together with the distribution of sulfamerazine between these fluids. The results make it possible to define more clearly the advantages of high as compared with low concentrations of sulfamerazine in body fluids in treatment of meningitis.

Although there is a possibility that sulfonamide therapy may in time be supplanted by penicillin for treatment of meningitis, the relative merits of the two agents remain to be evaluated. It is believed that the data here presented may be of assistance in making a comparison of the two.

\section{EXPERIMENTAL}

Through the cooperation of the staffs of the Medical and the Pediatrics Departments of the Philadelphia General Hospital, it was possible to observe the results of treatment of meningococcic meningitis with sulfamerazine in 188 patients. The observations extended over 18 months, and included periods in the winters of 1942-43 and of 1943-44 during which this disease was epidemic. Observations on 95 patients have already been reported (5), along with details concerning the management of the patients. Patients received intravenously 3 grams of sodium sulfamerazine and 1 gram orally of sulfamerazine on admission to the Fever Ward and subsequently, 1 gram orally of sulfamerazine every 4 hours. The only important departure from our previous methods of treatment has been the use of supplementary alkali therapy during 1943-44 for approximately half of the patients.

Cerebrospinal fluid was obtained by lumbar puncture either without local anesthetic or after infiltration with 1:1000 nupercaine. It was ascertained by test that nupercaine would not give color with the reagents used for determination of sulfamerazine. Many of the data in the literature on concentrations of sulfonamides in cerebrospinal fluid are valueless because of contamination of the fluids collected with procaine. The method of 
Bratton and Marshall (6) adapted to the KlettSummerson photoelectric colorimeter was used. Ultrafiltrates of serum were prepared at $37.5^{\circ} \mathrm{C}$. by the method of Lavietes (7). In this method, plasma or serum is filtered under pressure through a sheet of cellophane. A variable proportion of the sulfonamide, depending largely on the compound used, filters through the cellophane. That remaining is loosely combined with serum protein.

Blood serum or plasma was employed for analyses in preference to whole blood because the unequal distribution of sulfamerazine between cells and plasma introduces an additional variable, the concentration of cells, when whole blood is used. Concentrations of sulfamerazine in whole blood average approximately 0.7 of the plasma concentration (8), but the ratio may vary widely.

\section{RESULTS}

Relationship between concentration of sulfamerazine in plasma, plasma ultrafiltrate, and cerebrospinal fluid

The average daily concentration of sulfamerazine in blood serum or plasma and in cerebrospinal fluid simultaneously collected during treatment from a representative section of the group is shown in Figure 1. It will be seen that the concentration in serum approximated $16 \mathrm{mgm}$. per $100 \mathrm{ml}$. except for a transient rise during the fourth day. As with other sulfonamides, individual plasma and cerebrospinal fluid sulfamerazine differed widely, despite the fact that all patients received the same amounts. Variability of concentrations shown by each patient, however, was much less than that between different patients. The close dependence of cerebrospinal fluid on serum concentration is clear. Ratios of the two averaged 0.41 . A slight trend toward higher cerebrospinal fluid/plasma ratios as treatment progressed was not significant statistically. The relationship in individual patients between plasma sulfamerazine concentration and that in cerebrospinal fluid is shown in Figure 2. Despite the wide range of plasma concentrations represented, from 1.3 to $39.0 \mathrm{mgm}$. per $100 \mathrm{ml}$., the relationship remained the same at high and low levels. Some individuals differed markedly from the general trend, with cerebrospinal fluid/plasma ratios as high as 0.66 and as low as 0.19 . The highest ratios were observed in gravely ill patients and were explained by decrease in proteinbound sulfamerazine in plasma. Variations in protein-bound sulfamerazine of patients under these and other circumstances will be reported elsewhere.

Sulfamerazine concentration in cerebrospinal fluid is approximately 80 per cent of the concentration in ultrafiltrates of serum, as Figure 3 shows. If cerebrospinal fluid sulfamerazine concentration depended upon ultrafiltration alone, the values would fall along the diagonal, and departure therefrom represents the effects of the intervention of the tissues constituting the plasma/ cerebrospinal fluid barrier. Mainly, however,

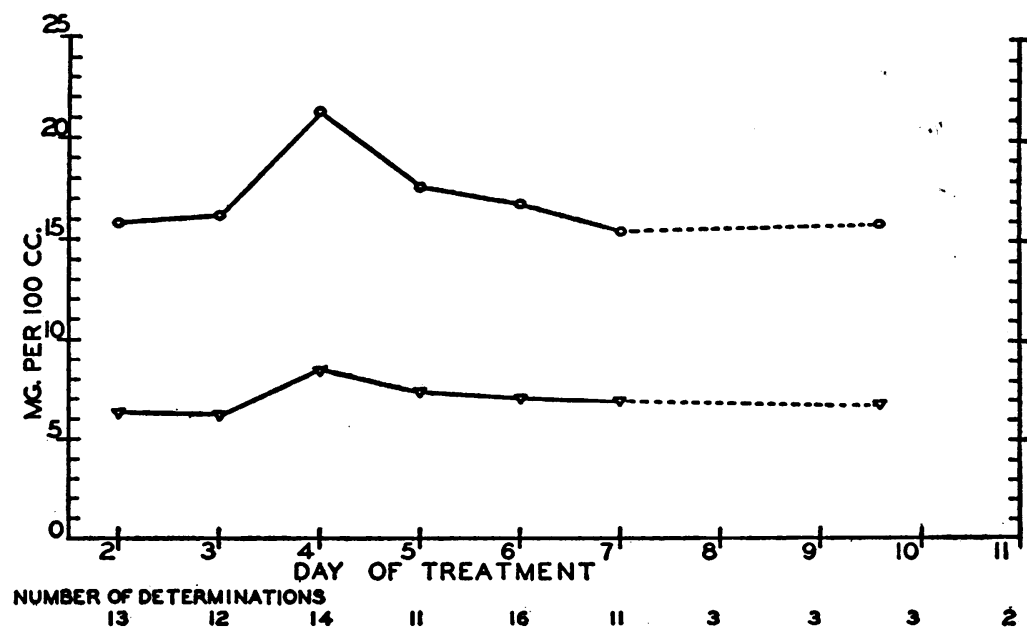

Fig. 1. Upper Curve: Average Serum Sulfamerazine Concentration During the Period of Treatment. Lower Curve: Average Concentration of Sulfamerazine in Cerebrospinal Fluid Collected Simultaneously 
concentration in cerebrospinal fluid is dependent upon the concentration of drug in plasma, and on such factors as plasma protein concentration and composition. These govern the proportion of the sulfamerazine that is combined with plasma protein and consequently is not ultrafilterable. Comparison of Figures 2 and 3 shows a diminution in scatter in Figure 3 as compared with Figure 2, and presumably much of the variation in cerebrospinal fluid/plasma ratio is due to variations in protein-bound sulfonamide. There was no change in relationship between sulfamerazine concentration in plasma ultrafiltrate and in cerebrospinal fluid in the course of treatment, nor did there appear to be any relationship between concentration of cerebrospinal fluid protein either to the preceding or to sulfamerazine concentration.

Relationship between concentration of sulfamerazine in body fluids and clinical response

The marked individual differences in average concentrations of sulfamerazine in body fluids during treatment apparently influenced the response to therapy. In Figure 4, the time required

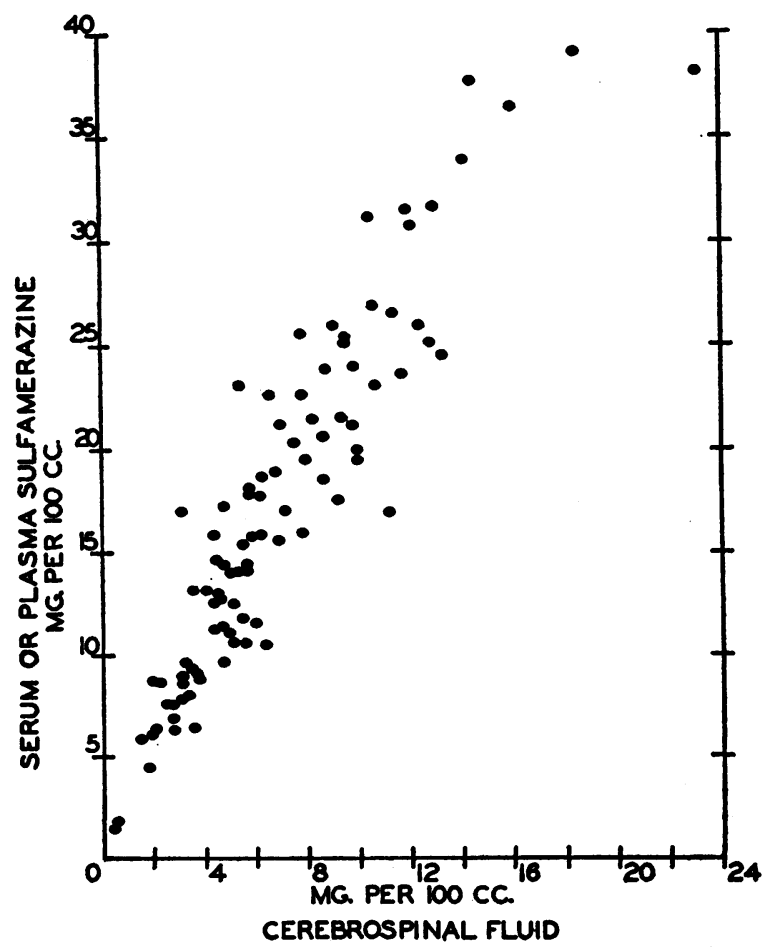

Fig. 2. Relationship Between Serum and Cerebrospinal Fluid Levels
$u_{\mathrm{MS} / \mathrm{inoc}}$

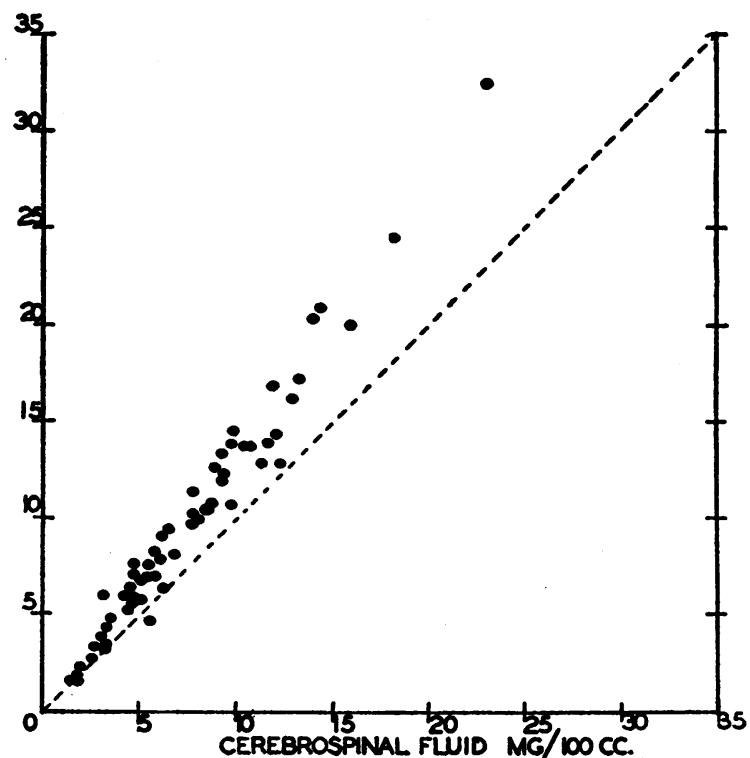

Fig. 3. Relationship Between Ultrafilterable Sulfamerazine of Plasma and Cerebrospinal Fluid Sulfamerazine

The diagonal represents the relationship to be expected if ultrafiltration alone determined the distribution.

for the patient's temperature (oral) to return to normal is compared with the average sulfamerazine concentration observed in the blood serum. In the presence of higher concentrations of sulfamerazine, the prevailing response was more rapid than that associated with lower concentrations. The relationship is approximated by a straight line. ${ }^{2}$ Although there were a number of patients

2 We have not attempted a detailed analysis of the type of curve that will best fit these data. Calculation of regression by the method of least squares yields a statistically valid regression coefficient $(p=<0.01)$. The coefficient of correlation $(r)=-0.43$. The corresponding relationship between temperature response and cerebrospinal fluid sulfamerazine (Figure 5) also was statistically significant $(p=<0.02) ;$ for the latter, $r=-0.44$. For ultrafiltrates these constants were $p=0.02$ and $r=-0.54$. All correlation coefficients were statistically significant but the differences were not.

The symbol $p$ refers, throughout this paper, to the evaluation of the probability of chance explaining the occurrence under consideration. When $p=0.05$ or less, the finding was considered significant. Methods described in Goulden (18) for evaluation of the significance of a regression coefficient were employed. 


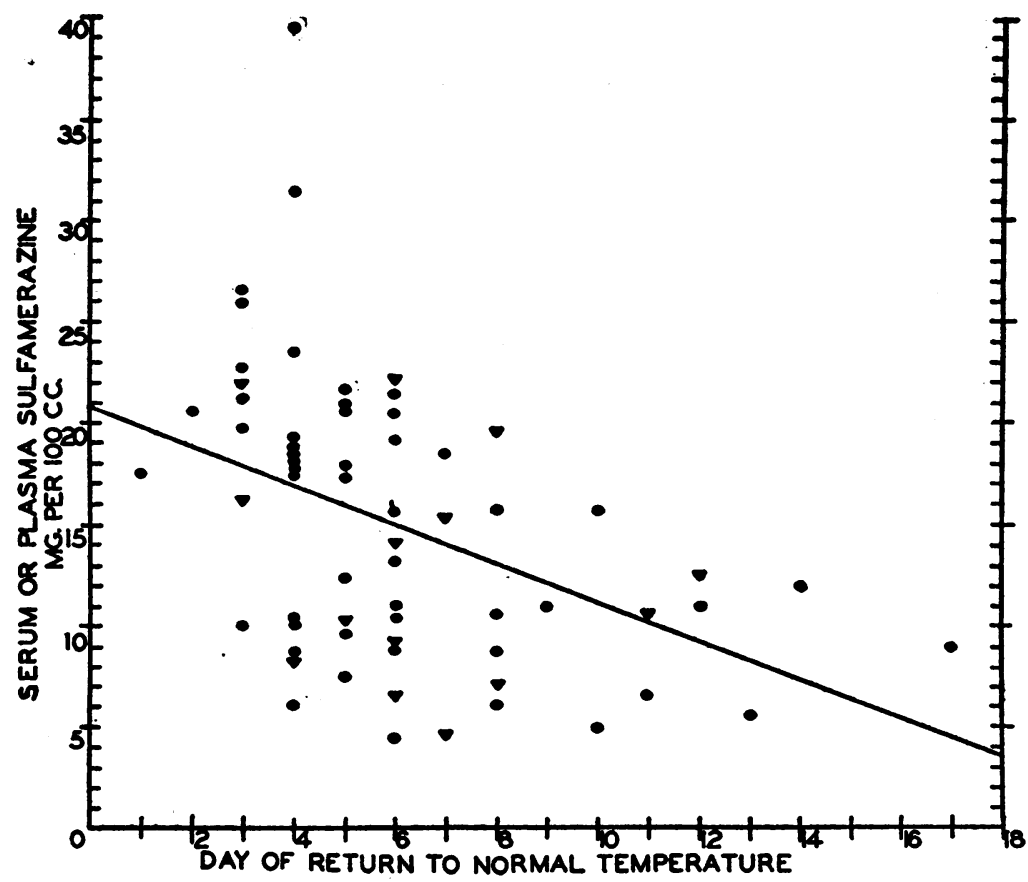

Fig. 4. Time Required After Starting Treatment for Patients to Become Afebrile as Related to Concentration of Sulfamerazine in Serum or Plasma

Triangles: Children under 12. Ovals: All others. Regression line in this and subsequent figures calculated by method of least squares.

with relatively low drug concentrations who responded rapidly to therapy, yet another considerable group showed low concentrations associated with a delayed response. Likewise, most individuals exhibiting high concentrations responded rapidly. Considering the response of the entire group, the average time required for return to normal temperature was shortened one day for each increment of $1 \mathrm{mgm}$. per $100 \mathrm{ml}$. in the serum sulfamerazine concentration. No difference is apparent between adults and children under 12 , nor between results obtained in $1942-43$ and 1943-44. A similar relationship when cerebrospinal fluid sulfamerazine concentration is substituted for that of plasma is shown in Figure 5.

In view of the belief that protein-bound sulfonamides do not possess bacteriostatic activity, it was deemed important to evaluate the correlation between ultrafilterable sulfamerazine and therapeutic action. The relationship shown in Figure 6 resembles closely that of plasma or cerebrospinal fluid. Coefficients of correlation are higher than those for the preceding comparisons but the dif- ference is not statistically significant. In each instance, high concentrations of sulfamerazine were associated with predominantly more favorable responses than were low concentrations.

Additional evidence relating the rapidity of response to drug level has been derived from evaluation of rate of disappearance of meningitic irritation (Figure 7). Here, the time required for its disappearance is plotted against the average concentration of drug. Again, the patients improving most rapidly were those exhibiting the higher concentrations of plasma sulfamerazine. Calculation of regression gave a statistically highly significant coefficient of regression. The relationship quantitatively is in good agreement with that observed for the response of temperature and that to be described for cerebrospinal fluid protein.

The decline in concentration of cerebrospinal fluid protein ${ }^{8}$ in the course of treatment with sulfamerazine also shows correlation with the

${ }^{8}$ Cerebrospinal fluid protein was determined by the photoelectric turbidimetric method of Looney and Walsk (19). 


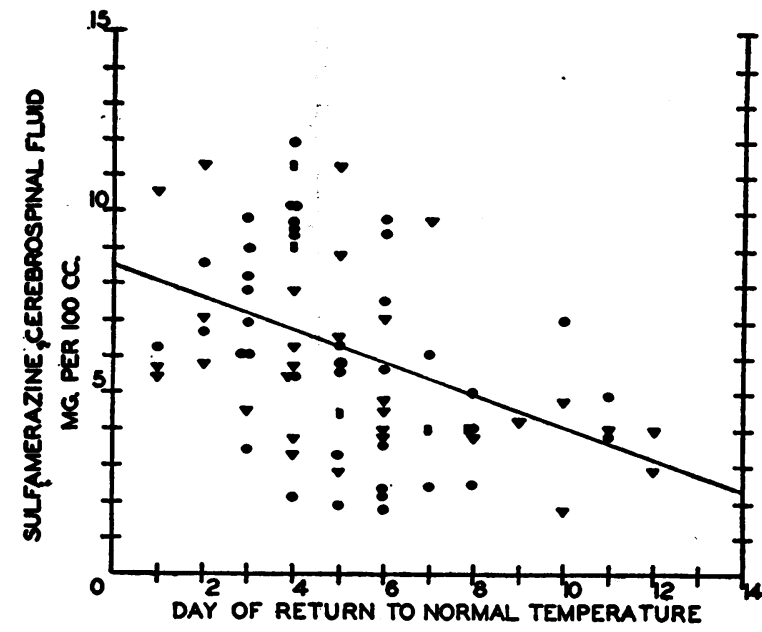

Fig. 5. Duration of Fever as Related to Concentration of Sulfamerazine in Cerebrospinal Fluid

Triangles: Patients studied in 1942-43. Ovals: $\mathrm{Pa}-$ tients studied in 1943-44. Squares: Samples obtained after temperature became normal.

level of sulfamerazine in plasma (Figure 8). Cerebrospinal fluid samples were collected soon after admission and again as opportunity occurred during treatment. When the concentration of sulfamerazine in plasma was high, the decrease in cerebrospinal fluid protein was more rapid than that occurring in the presence of lower concentrations of sulfamerazine in plasma. This relationship is obscured somewhat by the appreciable time interval between measurements of cerebrospinal fluid protein concentrations. Neverthe-

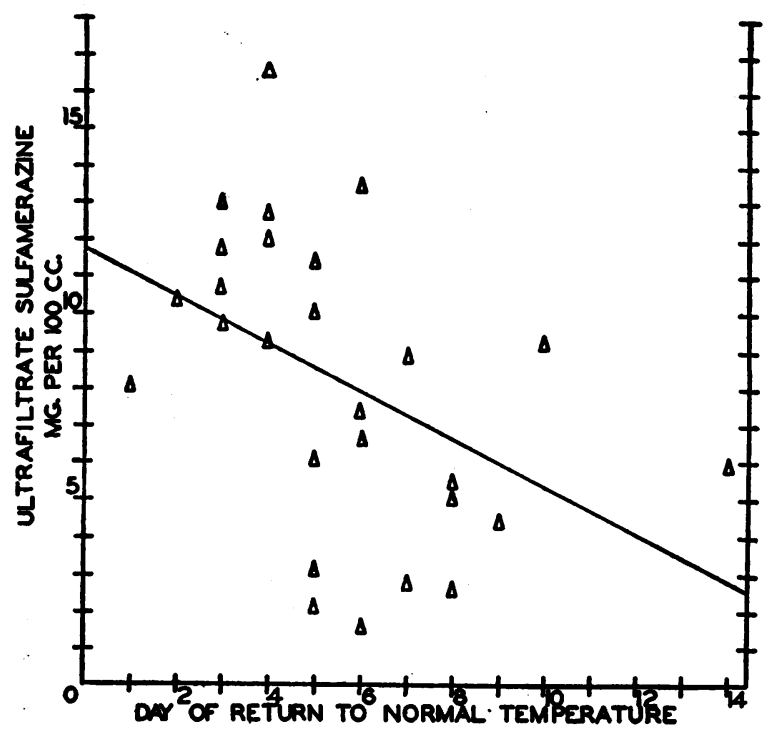

Fig. 6. Duration of Fever as Related to Concentration of Sulfamerazine in Plasma Ultrafiltrate

less, the relationship, tested statistically, yielded the regression line shown with $\mathrm{a}^{\prime}$ regression coefficient that was significant $(P=<0.02)$.

\section{TOXICITY}

Forty-six toxic reactions were noted in 40 of the 188 patients. Certain toxic effects of the sulfonamides are thought to be dependent upon the dosage and resulting concentration in body fluids. Since higher concentrations of sulfamerazine appear to be more effective therapeutically

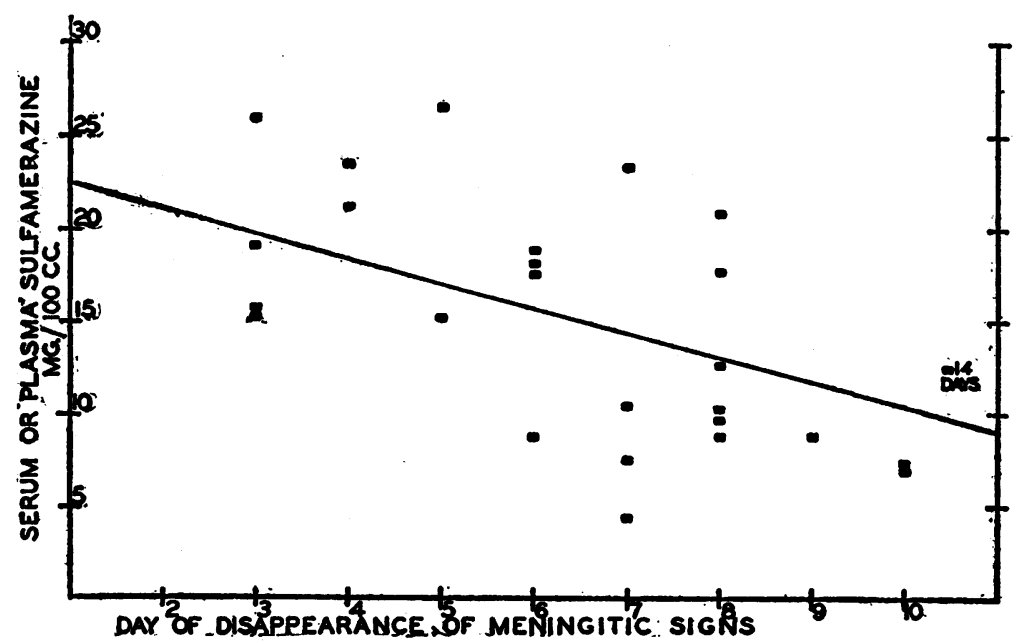

Fig. 7. Duration of Meningeal Irritation as Related to Concentration of Sulfamerazine in Serum or Plasma 


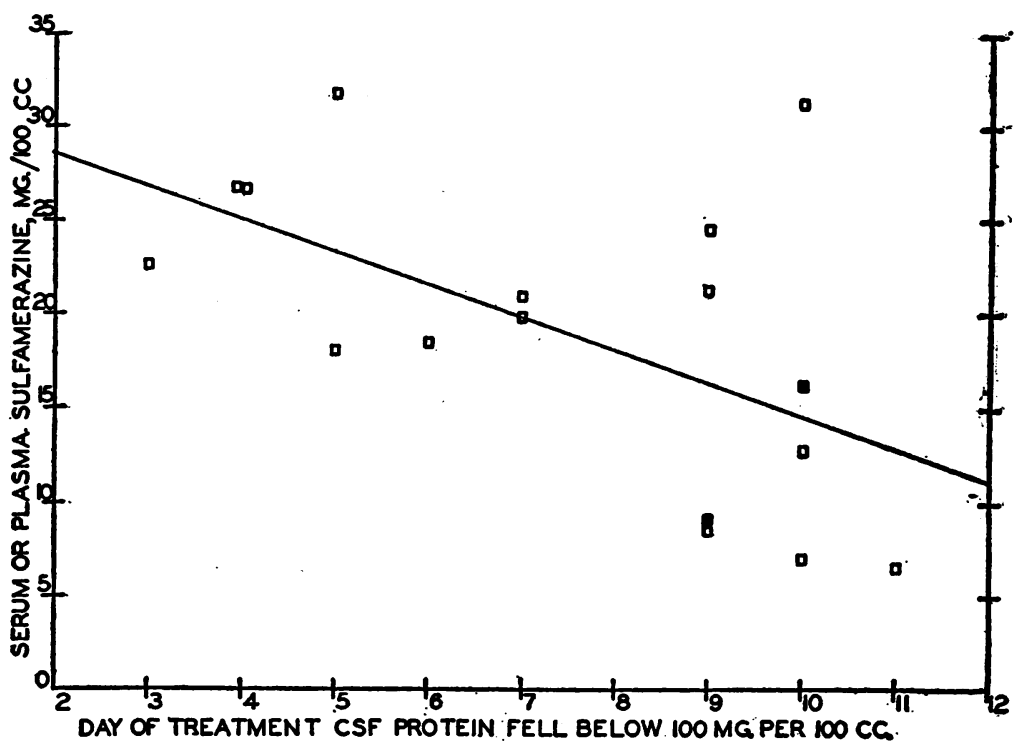

Fig. 8. Decrease in Concentration of Cerebrospinal Fluid Protein as Related to Concentration of Sulfamerazine in Serum or Plasma

Solid squares: Fatal cases; all others recovered.

than lower concentrations, it becomes desirable to evaluate the increased hazard of toxicity from the higher concentrations. Table I shows that such a relationship probably exists in regard to drug fever, since the frequency is much higher in the patients with concentrations in serum above the median level. Statistical analysis indicates ${ }^{4}$ that the relationship approaches the level accepted as significant. Drug rash, on the other hand, shows an incidence well removed from the level of significance, and there is no difference in frequency of hematuria at high and low concentrations in the serum. Certain investigators (9) have stated that renal damage from sulfonamides appeared to occur without any apparent relationship to quantity administered or level in blood.

The higher incidence of toxic effects associated with higher drug concentrations is offset in part by the increased frequency of such reactions as the duration of treatment increases. Figure 9 shows the occurrence of toxic manifestations of sulfamerazine during each day of the treatment period. Since the number of patients undergoing treatment decreased daily, the comparison. of early with later periods is best made in terms

4 The chi-square method with the Yates adjustment for continuity was employed. of the proportiion of patients undergoing treatment on any day who gave evidence of toxicity. Patients who may have shown more than one toxic reaction are included only once, at the time of the first reaction. The tabulation shows that occurrence of toxic manifestations is clearly dependent upon time and that the frequency rises after 8 days. The distribution appears to be bimodal, a peak occurring at the 5th day and again at the 9 th.

TABLE I

Incidence of toxic manifestations at higher and lower concentrations of sulfamerazine in plasma

\begin{tabular}{|c|c|c|c|c|}
\hline \multirow{2}{*}{\multicolumn{2}{|c|}{ Toxic manifestation }} & \multicolumn{2}{|c|}{ Plasma sulfamerazine } & \multirow[b]{2}{*}{ p } \\
\hline & & $\begin{array}{c}\text { Below } \\
14.6\end{array}$ & $\begin{array}{c}\text { Above } \\
14.6 t\end{array}$ & \\
\hline \multirow{2}{*}{$\begin{array}{l}\text { Microscopic } \\
\text { hematuria }\end{array}$} & Present & 6 & 5 & \multirow{2}{*}{$>0.5$} \\
\hline & Absent & 60 & 60 & \\
\hline \multirow{2}{*}{ Drug fever } & Present & 2 & 9 & \multirow{2}{*}{0.06} \\
\hline & Absent & 64 & 56 & \\
\hline \multirow{2}{*}{ Rash } & Present & 2 & 7 & \multirow{2}{*}{$>0.15$} \\
\hline & Absent & 64 & 58 & \\
\hline
\end{tabular}

† Median; mgm. per $100 \mathrm{ml}$. 

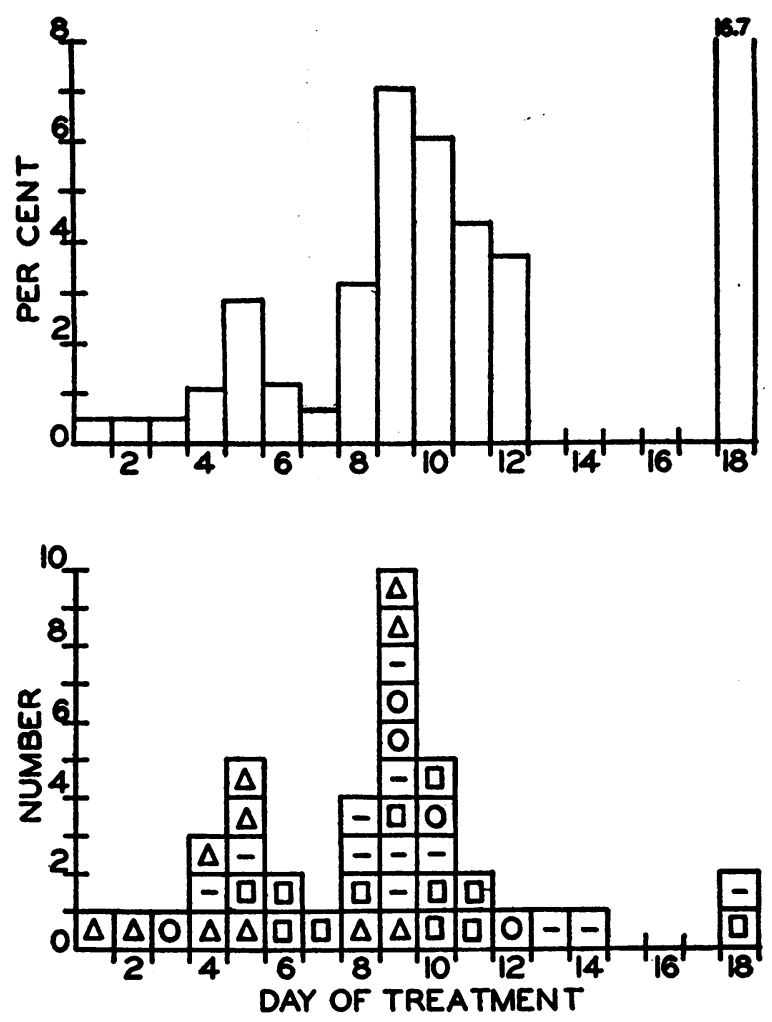

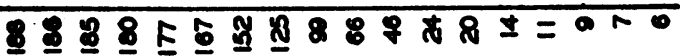
NUMBER OF PATIENTS UNDERGOING

Fig. 9. Incidence of Toxic Manifestations of SulfaMERazine as Related to Duration of Treatment

Lower histogram: Number of individuals showing evidence of toxicity each day after start of treatment. Nature of toxicity: Circles: gross hematuria; triangles: microscopic hematuria; squares: drug fever; dashes: drug rash.

Upper histogram: Incidence of initial toxicity adjusted for number of patients undergoing treatment each day. Since only the initial signs of toxicity are considered, entries in the lower histogram representing multiple reactions are omitted.

\section{Relationship of plasma sulfamerazine to mortality}

Plasma sulfamerazine concentrations of the patients who succumbed were $2.7,8.9,11.5,11.5$, $11.5,11.9,15.0,15.2,15.6,16.2,18.4$, and 24.4 mgm. per $100 \mathrm{ml}$. (average for each individual). The first obviously was undertreated and died 20 hours after therapy was started. The distribution of the others does not differ appreciably from that of the plasma sulfamerazine concentrations of patients that recovered.

\section{DISCUSSION}

The close relationship between sulfamerazine concentration in cerebrospinal fluid and in plasma ultrafiltrates shows that factors determining the ultrafilterability of sulfamerazine in serum are also predominantly those that govern concentration in the cerebrospinal fluid. In addition to the actual concentration of drug in plasma, these are the concentration of protein in plasma, and the chemical composition of the protein. Differences in the extent to which the various sulfonamides are combined with plasma protein appear to explain why cerebrospinal fluid sulfamerazine and sulfathiazole concentrations are less than half of the concentration in plasma, as compared with the much larger proportion of sulfanilamide and sulfadiazine. The two first mentioned are strongly bound to serum protein, the latter much less so.

The relative importance of sulfonamide levels in blood and cerebrospinal fluid in governing the effectiveness of these drugs has received considerable attention. For a time, sulfathiazole was thought to be unsuited for treatment of meningitis because of the low concentrations established in cerebrospinal fluid. However, it has been shown (4) that it was as effective as other sulfonamides for this purpose. Our results show that concentrations of a particular sulfonamide, sulfamerazine, in serum have as good a correlation with the patient's response as do the concentrations in cerebrospinal fluid. The same may be said of the correlation between therapeutic response and the concentration of drug in ultrafiltrates of serum. Thus, for evaluation of therapeutic effectiveness there appears to be insufficient advantage gained by measurement of drug concentration, either in cerebrospinal fluid or ultrafiltrate, as compared with serum or plasma, to warrant the additional effort required for the former. In view of the close relationship between the sulfamerazine concentrations in these fluids, and also in view of the current belief that meningococcic infection is a generalized infection and includes meningitis as only one manifestation, this result is not unexpected.

Our studies suggest that maintenance of high concentrations of sulfamerazine in plasma, 20 mgm. per $100 \mathrm{ml}$. or higher (equivalent to 14 mgm. or higher in whole blood), will lead to a 
more rapid therapeutic response than will lower concentrations. Higher concentrations will increase somewhat the incidence of drug fever and possibly rash and other manifestations of toxicity: However, these will be offset to some extent by the decreased duration of therapy, and resultant diminished hazard of toxicity, since the incidence of toxic reactions increases with time. Further studies, with larger dosages than those we have employed, are desirable to establish the optimal concentration range.

Data in the literature dealing with sulfamerazine are not sufficiently extensive to enable us to determine definitely whether the concentrationresponse relationship observed by us may be detected in similar studies made by others. Other workers (10) have presented figures for cerebrospinal fluid sulfamerazine concentrations of 14 patients and blood concentrations of 24 patients suffering from meningococcus infections. Satisfactory evaluation of the temperature response in relation to drug level is not possible, mainly because of the small groups studied, but partly because the use of whole blood rather than serum or plasma analyses impairs the value of the blood data for correlation studies. What may be evidence of a concentration-response relationship is discernible in their cerebrospinal fluid data. It should be noted that the delayed temperature fall in some of the patients may be explained by drug fever.

Opinion expressed in the recent literature is divided with respect to the merits of low as compared with higher dosages and serum levels of sulfadiazine in treatment of meningitic infections. There are numerous instances similar to that described by others (11) where a patient suffering from meningococcic meningitis failed to respond when the concentration in blood was low, 2.6 mgm., but improved promptly when this was raised to $7.5 \mathrm{mgm}$. per $100 \mathrm{ml}$. Bohan and Lusk (12) found average concentrations of 4 or $5 \mathrm{mgm}$. per $100 \mathrm{ml}$. of blood adequate for many patients, but advocated raising these to 12 or 14 if response was poor. Daniels, Solomon, and Jaquette (13) compared dosages giving average concentrations of 8.4 and $12.9 \mathrm{mgm}$. per $100 \mathrm{ml}$. in blood, respectively. The response of the meningitic infection was the same but toxicity was more marked in the group receiving the larger amounts. These authors state, however, that the comparison may have been influenced by diminishing severity of the disease while the smaller dosage was being tested. Applebaum and Nelson (14) also believe that moderate dosages of sulfadiazine are therapeutically adequate and present less hazard of toxicity. Marangoni and D'Agati (15), however, advocated maintenance of high concentrations (15 to $20 \mathrm{mgm}$. per $100 \mathrm{ml}$.) of sulfadiazine in treatment of meningococcic infection.

The relationship of the response to concentration is complex but some of the apparent inconsistencies can be clarified by referring to dosage-response relationships, shown by experimentally induced infections in animals, as described by Marshall, Litchfield, and White (16). With increasing intake and corresponding rise in blood levels of sulfapyridine or sulfanilamide, there was a marked improvement in response. The increased effectiveness per mgm. of rise in blood concentration of the drug gradually lessened, with a final tapering off of the response so that further increments of drug intake caused no additional decrease in mortality. A comparable relationship may be expected in man and the response to increasing concentrations of drug will depend upon the location of that increment on the concentration-response curve. The behavior of sulfamerazine as described in this study indicates that the concentrations encountered are on the ascending portion of such a curve. The evidence available for sulfadiazine suggests that concentrations in blood obtained by the authors previously mentioned probably are located on a plateau of a similar curve and that further moderate increases will not lead to commensurately improved response. Analogous curves relating rate of growth of bacteria to concentration of various sulfonamides in synthetic media, as described by Kohn and Harris (17) may show several plateaux characterized by slight gain in bacteriostasis per unit of increase interspersed between regions where the gain in bacteriostasis is pronounced. Such concentration-response relationships reflect the competitive combinations of the sulfonamides with the metabolic catalysts of the bacteria and depend not only upon the number of loci of action but also upon the presence and concentration of antagonists. Because of the complexity of the relationships involved and in view of the limited 
information available for sulfamerazine and sulfadiazine and for meningitic infections, it is not yet possible to correlate with any certainty the figures available for humans with such concentrationresponse curves. However, they do provide a guide as to the type of relationship to be sought.

Remaining to be considered is the possibility that the correlation we have observed between concentration of sulfamerazine in body fluids and clinical improvement may have been the result of a coincidental alteration of the pharmacodynamic behavior of the drug, resulting perhaps from illness of greater severity affecting the patients exhibiting low sulfamerazine concentrations, or other cause. All patients were receiving the same dosage, yet showed the marked differences in concentrations seen in Figures 2 and 4 . Age, incidence of complications, duration of illness, and other factors were comparable for the patients with high and those with low sulfamerazine concentrations. Sulfamerazine is not readily excreted by the kidney and it appears unlikely that differences in rate of excretion are responsible except that certain high values are so explained. Differences in absorption from the gastrointestinal tract thus must be the main cause of the differences in drug concentration. It is conceivable that some common factor such as nutritional deficiency might influence unfavorably both absorption of sulfamerazine and response to infection. That the behavior observed was not due to gradual decrease in concentration of sulfamerazine in the body fluids is shown in Figure 1, which shows that values are well sustained during the period in which the concentration-response relationship was observed. It seems probable, therefore, that differences in concentration of sulfamerazine in body fluids were primarily responsible for enhanced therapeutic effect in patients with high drug concentrations.

\section{SUM MARY}

The concentration of sulfamerazine in cerebrospinal fluid is approximately 80 per cent of that of an ultrafiltrate of plasma, and is closely dependent upon the same factors governing the concentration of the drug in the ultrafiltrate, i.e., concentrations of drug and protein in plasma, and composition of the protein.
In treatment of meningococcic meningitis, higher concentrations of sulfamerazine in body fluids were associated with more rapid return of body temperatures to normal, more rapid disappearance of meningitic irritation, and more rapid decrease in cerebrospinal fluid protein concentrationş, than were lower concentrations. For each milligram of increase in plasma sulfamerazine concentration, the average response was shortened by one day. The results suggest that the dosage employed and in common use is suboptimal, and that further trials with therapeutic concentrations in blood serum above $20 \mathrm{mgm}$. per $100 \mathrm{ml}$. are needed.

Determinations of concentrations of sulfamerazine in cerebrospinal fluid or plasma ultrafiltrates offer no advantage in evaluation of therapeutic response in meningococcic meningitis over such determinations in plasma.

Certain toxic manifestations (drug fever) of sulfamerazine appear to be more frequent at higher concentrations of drug in plasma. Others (rash, hematuria) showed no such relationship. The incidence of toxic manifestations increased appreciably after one week of treatment, reaching a maximum about 10 days after treatment was started.

\section{BIBLIOGRAPHY}

1. Smith, F. G., Sulfonamide Therapy in Medical Practice. F. A. Davis Co., Philadelphia, 1944.

2. Spink, W. W., Sulfanilamide and Related Compounds in General Practice. Year Book Publishers, Inc., Chicago, 1941.

3. Dingle, J. H., and Finland, M., Diagnosis, treatment, and prevention of meningococcic meningitis. War Med., 1942, 2, 1.

4. Banks, H. S., Sulphathiazole in cerebrospinal fever. Lancet, 1941, 1, 104.

5. Flippin, H. F., Reinhold, J. G., and Gefter, W. I., Sulfamerazine; clinical evaluation in 400 cases. M. Clin. North America, 1943, 27, 1447.

6. Bratton, A. C., and Marshall, E. K., Jr., A new coupling compound for sulfanilamide determination. J. Biol. Chem., 1939, 128, 537.

7. Lavietes, P. H., Anaerobic ultrafiltration. J. Biol. Chem., 1937, 120, 267.

8. Murphy, F. D., Clark, J. K., and Flippin, H. F., Studies on 2-sulfanilamido-4-methyl-pyrimidine (sulfamerazine, sulfamethyldiazine) in man. Am. J. M. Sc., 1943, 205, 717.

9. Murphy, F. D., Kuzma, J. F., Polley, T. Z., and 
Grill, J., Clinicopathologic studies of renal damage due to sulfonamide compounds: a report of 14 cases. Arch. Int. Med., 1944, 73, 433.

10. Hageman, P. O., Harford, C. G., Sobin, S. S., and Ahrens, R. E., Sulfamerazine: a clinical study of its pharmacodynamics, therapeutic value and toxicity. J. A. M. A., 1943, 123, 325.

11. Dingle, J. H., Thomas, L., and Morton, A. R., Treatment of meningococcic meningitis and meningococcemia with sulfadiazine. J. A. M. A., 1941, 116, 2666.

12. Bohan, J. L., and Lusk, F. B., Diagnosis and treatment of epidemic cerebrospinal meningitis. J. Lab. and Clin. Med., 1944, 29, 585.

13. Daniels, W. B., Solomon, S., and Jaquette, W. A., Meningococcic infection in soldiers. J. A. M. A., 1943, 123, 1.

14. Appelbaum, E., and Nelson, J., Sulfadiazine and its sodium compound in treatment of meningococcic meningitis and meningococcemia. Am. J. M. Sc., 1944, 207, 492.

15. Marangoni, B. A., and D'Agati, V. C., Treatment of 134 cases of meningococcic infection with massive doses of sulfadiazine. Am. J. M. Sc., 1944, 207, 67.

16. Marshall, E. K., Jr., Litchfield, J. T., Jr., and White, H. J., The comparative therapeutic activity of sulfanilamide, sulfapyridine, and diaminosulfone in streptococcus infections in mice. J. Pharmacol. and Exper. Therap., 1940, 69, 89.

17. Kohn, H. I., and Harris, J. S., On the mode of action of the sulfonamides. I. Action on escherichia coli. J. Pharmacol. and Exper. Therap., 1941, 73, 343.

18. Goulden, C. H., Methods of Statistical Analysis. John Wiley and Sons, New York, 1939.

19. Looney, J. M., and Walsh, A. I., The determination of spinal fluid protein with the photoelectric colorimeter. J. Biol. Chem., 1939, 127, 117. 